\title{
Inhalt, Vol. 3, No. 2, 1980
}

\section{Contents}

Impressum 47

Bombik, B. M.; Oertel, J.; Schneider, D,; Gerhartz, H.,

Berlin

Chemotherapie des metastasierten Kolonkarzinoms mit

Nitroseharnstoffen 49

Haas, R. J.; Helmig, M.; Prentl, E., München

Effektivität intramuskulär verabreichten Cytosin-Ara-

binosids bei der ambulanten Behandlung von Kindern

mit akuter Leukämie in Remission 53

Havers, W.; Stolímann, B.; Bachmann, H. J.; Bamberg, M.; Stambolis, C, Essen

Bestrahlungsbedingte Nierenerkrankung nach Behand

lung von Malignomen im Kindesalter

58

Sauer, Hansjörg; Schalhorn, Andreas, München

Rationale Grundlagen und Praxis des Citrovorumfaktor-

(Leucovorin ${ }^{\circledR}$ )-Schutzes nach hochdosierter Metho-

threxat ${ }^{\circledR}$-Therapie 64

Schmitt, G.; Scherer, Ë., Essen

Neutronentherapie am Radiologischen Zentrum des

Westdeutschen Tumorzentrums des Universitätsklini-

kums Essen 72

Sellschopp-Rüppell, Almuth, Heidelberg

Psychologische Betreuung von onkologischen Patienten . 74

Siemers, E.; Seeber, S.; Höffken, K.; Schmidt, C. G.,

Essen

Ergebnisse der zytostatischen Therapie mit Cyclophos-

phamid, Vincristin, Adriamycin und DTIC (CYVADIC)

bei lokalisierten und metastasierten Osteosarkomen -

Eine retrospektive Analyse 78

Hinweise für Autoren $\quad 52$

Letzte Meldungen 62

Buchbesprechungen $\quad 85$

Onkologie-Mitteilungen $\quad 87$

Imprint 47

Bombik, B. M.; Oertel; J.; Schneider, D.; Gerhartz, H,,

Berlin

Chemotherapy Of Advanced Colorectal Cancer with

Nitrosoureas 49 
Haas, R. J.; Helmig, M.; Prentl, E., München

Effect on Peripheral Blood Cells in Children with Acute

Leukemia During Remission by Intramuscular Injection

of Arabinosyl-Cytosine 53

Havers, W.; Stollmann, B.; Bachmann, H. J.;

Bamberg, M.; Stambolis, C, Essen

Radiation-Induced Renal Diseases after Therapy of

Malignant Tumors in Childhood

58

Sauer, Hansjörg; Schalhorn, Andreas, München

Rationale and Practice of the Citrovorum Factor Rescue

after High-Dose Methotrexate Therapy

64

Schmitt, G.; Scherer, E., Essen

Neutron Therapy at the Department of Radiotherapy,

Westdeutsches Tumorzentrum, Universitätsklinikum

Essen 72

Sellschopp-Rüppell, Almuth, Heidelberg

Psychological Care of Oncological Patients 74

Siemers, E.; Seeber, S.; Höffken, K,; Schmidt, C. G.,

Essen

Cyclophosphamide, Vincristine, Adriamycin and DTIC

(CYVADIC) in localized and metastatic osteogenic

sarcomas. A retrospective analysis $\quad .78$

Instruction for Authors 52

Last News $\quad 62$

Book Reviews $\quad 85$

Oncology Communications $\quad .87$

Bibliographischer Hinweis: Inhaltsverzeichnisse dieser Zeitschrift erscheinen regelmäßig in current contents ${ }^{\circledR}$ sowie in anderen bibliographischen Diensten. 